\title{
المناهج ومهارات القرن الحادي والعشرين \\ Curricula and skills of the twenty-first century
}

$$
\text { أ.د. محمد أمين المفتي }
$$

أستاذ المناهج وطرق تدريس الرياضيات

كلية التربية - جامعة عين شمس ولتربي الرياضيات

elmofti44@hotmail.com 
هدفت هذه الورقة البحثية إلقاء الضـوء على مهارات القرن الحادي والعشرين، وأهيتها في المناهج الدراسية، وقد تم عرض لمحة تاريخية عن تطور تلك المهارات إلي أن تم تصنيفها إلي ثلاث مهارات أساسية هي: مهارات التعلم والابتكار ، والمهارات المهنية والحياتية، ومهارات محو الأمية الرقمية.

وبالرغم من تضمين أهداف المناهج الحالية لمهارات القرن الحادي والعشرين والتأكيد على ضرورة إتقان

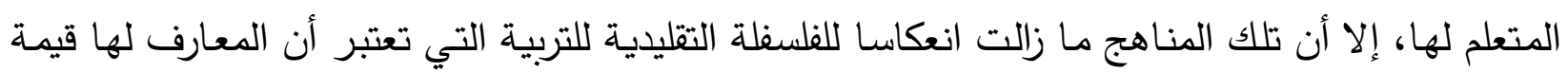

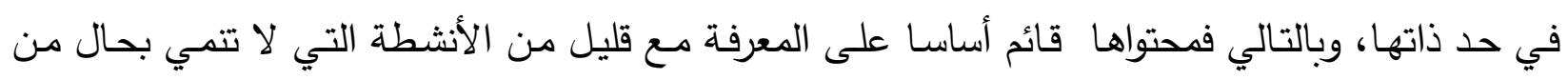

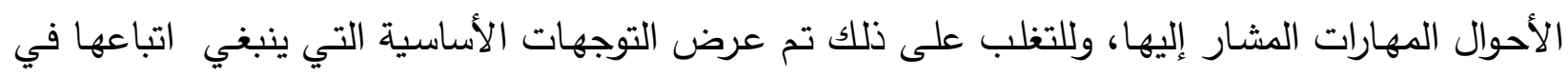
بناء المناهج لإكساب المتعلم هذه المهارات وتتميتها. الكلمات المفتاحية: (المناهج الدراسية، مهارات القرن الحادي والعشرين).

\section{Abstract:}

This research paper attempted to focus on twenty-first-century skills and their importance in school curricula. A historical overview of these skills' development was presented. They were classified into three basic skills: learning and innovation skills, professional and life skills, and digital literacy skills. Although twenty-first-century skills were included in the current curriculum objectives and there is an emphasis on their necessity for the learner, these curricula are still a reflection of the traditional philosophy of education. This philosophy considers that knowledge has value in itself. Therefore the content is based mainly on knowledge with few activities that are not related to twenty-first-century skills. To overcome this problem, the basic approaches that should be followed in building curricula were presented to provide the learner with these skills and their development

Keywords: School -Curricula-Twenty first century- skills 


\section{"المناهج ومهارات القرن الحادي والعشرين"}

\section{لمحة تاربخية:}

أجرت مجموعة من المؤسسات الحكومية، ومؤسسات الرأي والفكر، والهيئات البحثية في السنوات الأولى

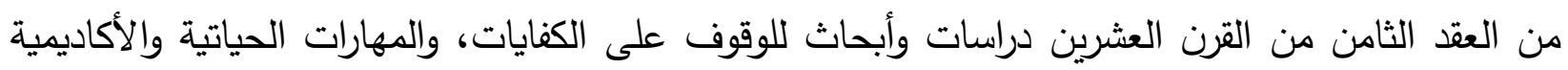
التي ينبغي أن يمتلكها الفرد في المستقبل كي يواصل تعليمه وعمله بنجاح، وتحديدا في القرن الحادي والعشرين، وقد تم التوصل إلى هذه الكفايات والمهارات.

بدأ التطبيق في التعليم والعمل في عدة دول منها الولايات المتحدة، وكندا، وإنجلترا، ونيوزيلاند، وفي

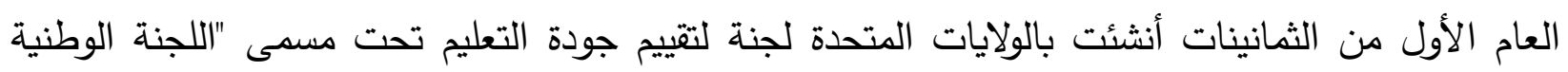

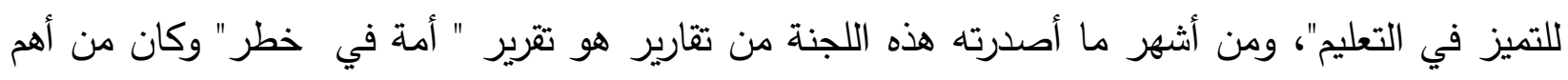

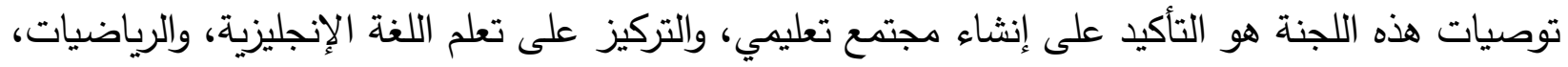
والعلوم، والدراسات الاجتماعية، والكمبيوتر .

وركزت نظم التعليم ومناهجه في معظم دول العالم حتى بداية القرن الحادي والعشرين على أهمية المعرفة وقيمتها في حد ذاتها، وبالتالي تكدست المناهج بالمعلومات والمعارف التي تهدف إلى تتمية المهارات

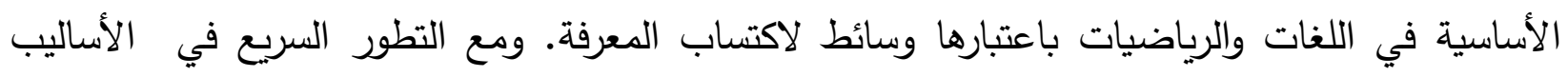

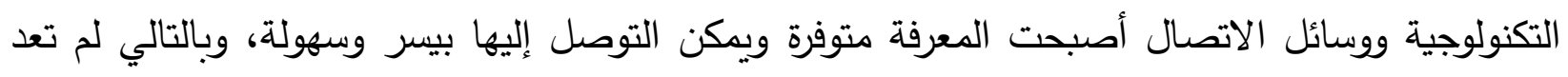
للمعرفة في حد ذاتها أو للمهارات الأساسية نفس القيمة السابقة.

وظهرت الحاجة إلى مهارات أخرى مثل مهارات العمل الجماعي، وحل المشكلات، والتواصل مع الآخرين، والتفكير الناقد للنجاح في التعلم والعمل في القرن الحادي والعشرين. إطلالة على مهارات القرن الحادي والعشرين:

يوجد اتفاق بين المؤسسات الحكومية، ومؤسسات الرأي والفكر، والهيئات البحثية على أنه يمكن تصنيف

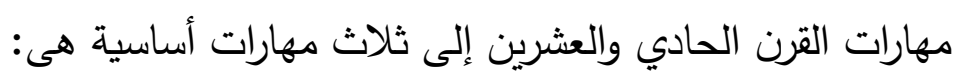
1- - 20 - مهارات التعلم والابتكار وتتضدن التفكير الناقد، والتفكير الابتكاري، وحل المشكلات، والتفكير في التفكير 
العدد الثاني

مارس 2021
المجلة الدولية للمناهج والتربية التكنولوجية

\section{IJCTE}

\section{2- المهارات المهنية والحياتية}

وتتضمن التواصل والتعاون، المبادرة، والتوجيه الذاتى، والمرونة، والتفاعل الاجتماعى والثقافي، الإنتاجية، والمساءلة، والطلاقة التكنولوجية

$$
\text { 3- مهارات محو الأمية الرقمية }
$$

وتتضمن محو الأمية المعلوماتية، والأمية في التكنولوجيا والاتصالات، ومحو الأمية الإعلامية والسؤال الآن : هل المناهج الحالية تعمل على إكساب المتعلم هذه المهارات؟

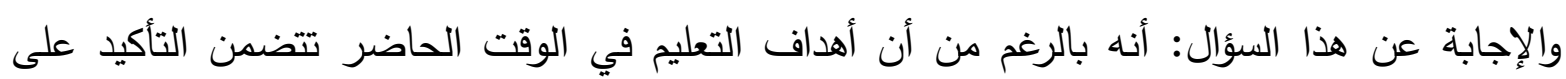

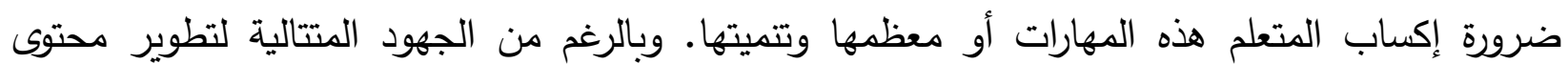

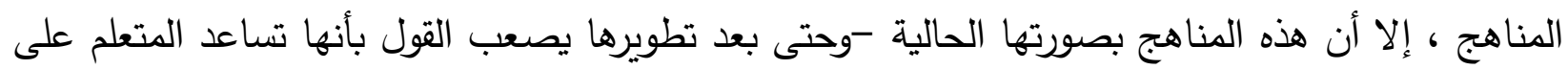

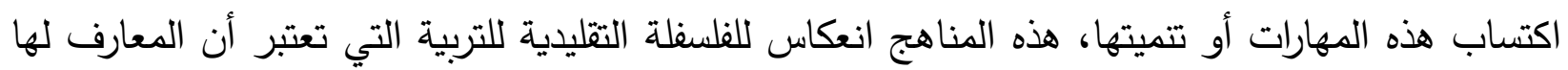

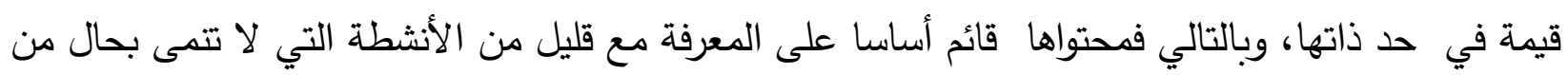

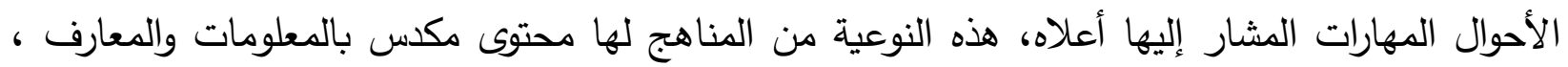

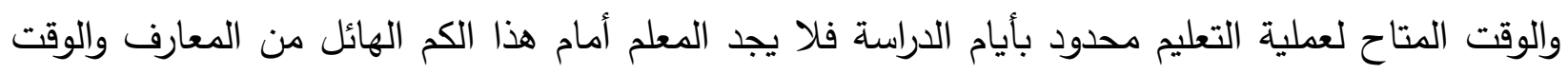

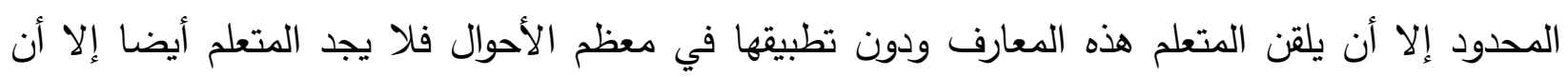

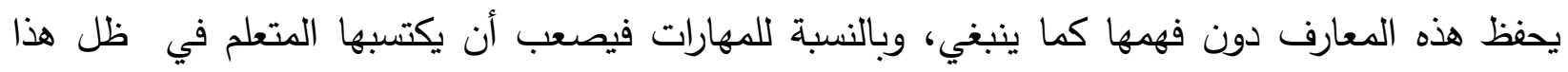
التظيم المنهجي القائم أساسا على المعرفة.

يتبع هذا السؤال من الناحية المنطقية سؤال آخر مفاده: ما التوجهات الأساسية التي ينبغي اتباعها في بناء المناهج لإكساب المتعلم هذه المهارات وتتميتها؟ التوجهات الأساسية: - 20 - 2 بالنسبة للأهداف:

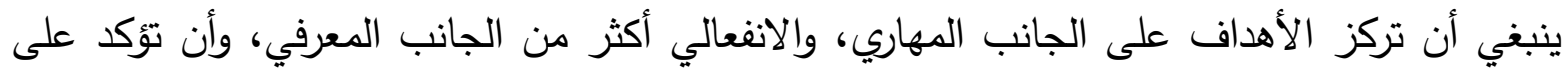

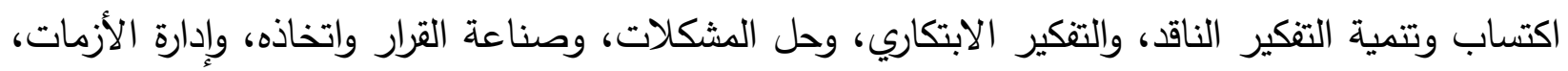

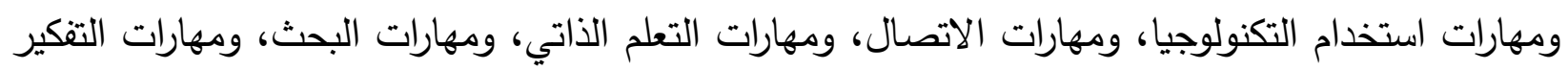
الجماعي. 
بالنسبة للمحتوى:

التخفف من المعارف والمعلومات السردية، والتركيز على المعلومات الموظفة، والمفاهيم الكبرى، والمواقف المشكلة، والأنشطة الجماعية والمشروعات. تضمين موضوعات عن البيئة والثقافات الأخرى وحياة الشعوب، والتكنولوجيا، وتعليم التفكير •

تظيم المحتوى : ينظم المحتوى في صورة مواقف مشكلة سواء أكاديمية أو حياتية، ومواقف للمناقشة والحوار ، ومشروعات جماعية مع مراعاة التكامل بين الأنساق المعرفية بقدر الإمكان. بالنسبة لإستراتيجيات التعليم والتعلم:

تستخدم مجموعة من إستراتيجيات التعليم والتعلم التي تتناغم وتتسق مع المحتوى لتحقيق الأهداف المشار إليها، وتتمثل في أسلوب حل المشكلات، والتعلم التشاركي، والتعلم التعاوني، والتعلم الذاتي،

Contracting. والاكتشاف بأنواعه والتعلم بالتعاقد بالنسبة للأساليب التكنولوجية: توظف الأساليب التكنولوجية المناسبة لتعليم المحتوى وتعلمه في كل المواقف التعليمية / التعلمية بالنسبة للتقويم: تستخدم الأسئلة البحثية، والأسئلة التباعدية، والموقف المشكلة (أكاديمية - حياتية)، وأسئلة تقيس اكتشاف الأنماط، وتكوين العلاقات، والاستتتاج، وأسئلة تقيس القدرة على الذهاب إلى ما وراء المعلومات المتاحة. 